\title{
Uma Contribuição ao Estabelecimento de Características de Qualidade para Aplicações Educacionais Móveis
}

\author{
Gustavo W. Soad ${ }^{1}$, Nemésio F. Duarte Filho ${ }^{1}$, Ellen F. Barbosa ${ }^{1}$ \\ ${ }^{1}$ Instituto de Ciências Matemáticas e Computação - Universidade de São Paulo (USP) - \\ São Carlos - SP - Brasil \\ \{gustavo.soad\}@usp.br, \{nemesio, francine\}@icmc.usp.br
}

\begin{abstract}
Resumo. A crescente utilização de aplicações educacionais móveis permite que alunos e professores tenham facilidades na execução de atividades e práticas educacionais. Não há, entretanto, um método adequado para avaliar a qualidade deste tipo de aplicação, já que os utilizados para tal são genéricos e não contemplam os aspectos específicos ao contexto pedagógico e móvel. Nesse cenário, o presente trabalho apresenta um conjunto de características de qualidade para aplicações educacionais móveis, considerando os aspectos técnicos, pedagógicos, socioeconômicos e socioculturais, identificadas a partir de uma revisão sistemática.
\end{abstract}

\begin{abstract}
The increasing use of mobile learning applications allows students and teachers to have facilities in the execution of activities and educational practices. There is no, however, an appropriate method to evaluate the quality of this type of application, since used for that are generic and do not include the specifics of the pedagogical and mobile context. In this scenario, this paper presents a set of quality characteristics for mobile learning applications, considering the technical, educational, socioeconomic and sociocultural aspects, identified from a systematic review.
\end{abstract}

\section{Introdução}

Nos últimos anos, temas relacionados ao ensino e aprendizagem têm sido amplamente discutidos e estudados pela comunidade científica. Em especial, aplicações computacionais têm apresentado uma crescente importância, desempenhando um papel fundamental em atividades de ensino e aprendizagem, sendo relevantes não apenas no âmbito acadêmico como também no meio industrial [Barbosa 2004, Svetlana et al. 2009].

Tais aplicações proporcionam, entre várias práticas educacionais inovadoras, uma nova modalidade de ensino, definida como aprendizagem móvel ou mobile learning (m-learning) [Kinshuk et al. 2003, Nah et al. 2008, O'Malley et al. 2003, Wexler et al. 2008]. Em linhas gerais, m-learning se caracteriza por proporcionar uma forte interação entre aprendizes e tutores, possibilitando que ambos contribuam, participem e acessem as práticas educacionais de ensino por meio de dispositivos móveis (celulares, tablets, laptops, rádio, televisores, entre outros) a qualquer hora e em qualquer lugar.

Apesar dos benefícios proporcionados no contexto de ensino e aprendizagem, por se tratar de um conceito novo e ainda incipiente, o m-learning apresenta problemas e desafios organizacionais, culturais e tecnológicos em sua construção e utilização. Entre 
os problemas existentes, está a avaliação de qualidade desse tipo de aplicação, já que os métodos existentes são genéricos e, por este motivo, não contemplam os aspectos específicos das aplicações m-learning.

Aspectos de qualidade, em particular, representam um aspecto importante a ser abordado, principalmente em virtude da crescente popularidade dos aplicativos móveis em diferentes setores da sociedade. Neste cenário emergente, a qualidade não é apenas relacionada a aspectos técnicos. Há também uma necessidade de lidar com questões intrínsecas (por exemplo, educacional, sociocultural e econômica) relacionadas com as atividades diárias de aprendizes e tutores [Economides 2008]. Poucos estudos sobre as diretrizes de qualidade para aplicações móveis são encontrados na literatura. Como consequência, torna-se difícil identificar mecanismos bem definidos e amplamente utilizados para apoiar a avaliação da qualidade de aplicações educacionais móveis.

Embora existam na literatura vários modelos de qualidade, como os apresentados na norma ISO/IEC 9126 [ISO/IEC 9126 2001] e ISO/IEC 25010 [ISO/IEC 25010 2010], propostos pela ISO (International Organization for Standardization), tais modelos são genéricos e possuem deficiências ao lidar com aplicações m-learning. Por exemplo, os aspectos técnicos deste tipo de aplicação são diferentes em relação a outras aplicações, além de outras deficiências observadas. De modo geral, um modelo de qualidade para este tipo de aplicação também deveria suprir os aspectos pedagógicos, socioeconômicos e socioculturais [Economides 2008].

Nesse contexto, este trabalho tem como principal objetivo identificar um conjunto de características de qualidade para aplicações m-learning, considerando os aspectos técnicos, pedagógicos, socioeconômicos e socioculturais, além de identificar também as características dos dispositivos móveis que possam influenciar na qualidade de uma aplicação m-learning.

$\mathrm{O}$ artigo encontra-se organizado como se segue. A Seção 2 apresenta a fundamentação teórica sobre aprendizagem móvel. A Seção 3 descreve o planejamento e a condução da revisão sistemática utilizada para identificar as características relacionadas à qualidade. A Seção 4 traz os resultados obtidos e apresenta uma análise sobre eles, além de fazer a proposta de um catálogo de características. Por fim, na Seção 5, são sumarizadas as conclusões do trabalho e as perspectivas para continuidade de pesquisa.

\section{Fundamentação Teórica}

Nesta seção, são apresentados os conceitos relacionados a aprendizagem móvel e a qualidade de software, com o intuito de contextualizar o estudo realizado.

\subsection{Aprendizagem Móvel (m-learning)}

Aprendizagem móvel ou m-learning, é uma modalidade de aprendizagem que, por meio de dispositivos móveis, permite o ensino e a aprendizagem sem limitações de tempo e espaço.

M-learning também pode ser definido como qualquer tipo de ensino ou aprendizagem que ocorre quando o aluno não está em algum lugar predeterminado ou fixo, ou quando o indivíduo aproveita-se de oportunidades de aprendizagem disponibilizadas pelas tecnologias móveis, associando assim os conceitos de tecnologia e mobilidade [O’Malley et al. 2003]. 
A utilização de aplicações m-learning permite aos indivíduos serem mais produtivos quando consomem, criam ou interagem com as informações, por meio de dispositivos móveis e portáteis, que acompanham o indivíduo de forma regular, do início ao fim das tarefas [Wexler et al. 2008]. Em outra perspectiva relacionada, $m$-learning pode ser definido como a capacidade do uso de dispositivos portáteis para acesso a recursos de aprendizagem [Kinshuk et al. 2003].

Alguns dos principais benefícios dos dispositivos móveis vão muito além de acessibilidade, comodidade e comunicação. Com dispositivos móveis os aprendizes podem utilizar aplicativos (processamento de texto, fotos), ambientes específicos de aprendizagem, acesso a Web, ferramentas de colaboração, redes sociais, e-books, entre outros.

Com a evolução da tecnologia e inclusão digital, fica clara a relevância de $m$ learning para sociedade atual. Os benefícios oferecidos pelo m-learning estão além da acessibilidade, comodidade e comunicação. Por meio de dispositivos móveis, os aprendizes podem utilizar ambientes específicos de aprendizagem, ter acesso a Internet, ferramentas de colaboração, redes sociais, $e$-books, entre outros.

Atualmente existem várias pesquisas sobre os benefícios que a aprendizagem móvel pode trazer, bem como sobre as limitações encontradas na sua utilização. Estudos sugerem que a utilização da aprendizagem móvel desperta o interesse do aluno, estimula a interação social provendo mecanismos de comunicação entre os usuários da aplicação, além de estimular a discussão sobre os materiais dos cursos [Huang et al. 2010, Mishra and Chavhan 2012, Picek and Grcic 2013, Fazlina et al. 2013, Al-Ani et al. 2013].

\subsection{Qualidade de Software}

A qualidade está representada na Engenharia de Software pela área conhecida como Qualidade de Software. Esta área é responsável por garantir a qualidade dos produtos de software por meio de processos definidos. A qualidade de software não aparece simplesmente "do nada". Ela é o resultado de um bom gerenciamento de projeto e uma prática consistente de Engenharia de Software [Pressman 2010]. Ainda segundo Pressman (2010), a qualidade de software pode ser definida como a conformidade a requisitos funcionais e de desempenho explicitamente declarados, a padrões de desenvolvimento claramente documentados e a características implícitas que são esperadas de todo software profissionalmente desenvolvido.

A qualidade de software também pode ser entendida como um conjunto de características a serem satisfeitas em um determinado grau, de modo que o produto de software atenda às necessidades implícitas e explícitas de seus usuário [ISO/IEC 8402 1994]. Nesse contexto, as necessidades implícitas são aquelas que não são expressas, porém são necessárias para se ter o produto final. Já as necessidades explícitas são especificadas em documentos para serem atendidas pelo produto de software, ou seja, são necessidades definidas pelos interessados no produto a ser desenvolvido.

A avaliação de qualidade de um produto de software pode ser avaliada sob os aspectos do processo de desenvolvimento, que se refere às atividades realizadas durante as etapas do seu ciclo de vida, e sob a qualidade de produto, que se refere às características do produto após o seu desenvolvimento. 


\section{Revisão Sistemática}

Uma revisão sistemática é um meio de identificar, avaliar e interpretar todas as pesquisas relevantes disponíveis e relacionadas com uma questão de pesquisa, tema ou fenômeno [Kitchenham and Charters 2007].

A condução de uma revisão sistemática envolve um processo composto por seis etapas: (i) definição das questões de pesquisa; (ii) definição da string de pesquisa; (iii) definição dos critérios de inclusão e exclusão; (iv) seleção dos estudos primários relevantes; (v) extração dos dados; e (vi) síntese dos resultados.

Nesta seção são detalhadas as etapas de planejamento e condução de uma revisão sistemática no contexto de ambientes m-learning, além de sintetizar os resultados obtidos. Outros detalhes sobre a revisão são apresentados em [Soad et al. 2015].

\subsection{Planejamento da Revisão Sistemática}

O objetivo da pesquisa foi identificar características de qualidade relacionadas a aplicações m-learning e, caso existam, identificar as métricas relacionadas a elas. As características foram divididas em quatro categorias: (i) técnicas, (ii) pedagógicas, (iii) socioeconômicas, e (iv) socioculturais.

Como a qualidade da aplicações pode ser afetada pelos dispositivos móveis, a revisão sistemática conduzida, também incluiu em seus objetivos, a identificação das características dos dispositivos que possam afetar a qualidade de uma aplicação m-learning .

Para atingir os objetivos, foram estabelecidas cinco questões de pesquisa, apresentadas na Tabela 1. A partir da definição das questões de pesquisa, foram estabelecidas as estratégias de busca utilizadas na recuperação dos estudos, como as fontes de busca, idioma dos textos, palavras-chave e os critérios de inclusão e exclusão, que são apresentados na Tabela 2.

Tabela 1. Questões de Pesquisa

QP1 - Quais são as características dos dispositivos móveis que podem afetar a qualidade das aplicações m-learning? QP2 - Quais são as características técnicas relacionadas à qualidade das aplicações $m$-learning?

QP3 - Quais são as características pedagógicas relacionadas à qualidade das aplicações m-learning?

QP4 - Quais são as características socioeconômicas relacionadas a qualidade das aplicações m-learning?

QP5 - Quais são as características socioculturais relacionadas à qualidade das aplicações m-learning?

Tabela 2. Critérios de Inclusão e Exclusão

\begin{tabular}{l|l}
\hline Critérios de Inclusão & Critérios de Exclusão \\
\hline $\begin{array}{l}\text { Estudos relacionados a m-learning e que apresentam carac- } \\
\text { terísticas de qualidade. }\end{array}$ & $\begin{array}{l}\text { Estudos primários que evidenciam características e requisitos } \\
\text { de ambientes } m \text {-learning sem embasamento teórico ou prático. }\end{array}$ \\
\hline $\begin{array}{l}\text { Estudos que apresentam métricas de qualidade relacionadas a } \\
\text { m-learning. }\end{array}$ & $\begin{array}{l}\text { Estudos primários que estejam em idiomas diferentes de } \\
\text { inglês e português, dificultando a coleta e a identificação de } \\
\text { informações. }\end{array}$ \\
\hline Estudos relacionados a avaliação de qualidade para m-learning & Estudos não disponíveis, que não puderam ser recuperados. \\
\hline
\end{tabular}

As fontes de busca escolhidas para a pesquisa foram: IEEEXplorer, ACM, Science Direct, Scopus e Web of Science. Elas foram selecionadas de acordo com as principais bases de pesquisa que indexam estudos relacionados a Ciências da Computação.

Além das fontes de busca apresentadas, também foram realizadas buscas nos seguintes periódicos: Journals Computer \& Education, IEEE Transactions on Learning, 
ACM Transactions on Computing Education, International Journal on E-Learning, Ubiquitous Learning: An International Journal e International Journal of Interactive Mobile Technologies. Também foram realizadas buscas nas conferências: Mobile Learning Conference, Simpósio Brasileiro de Qualidade de Software (SBQS) e International Conference on Quality Software.

\subsection{Condução da Revisão Sistemática}

Para a realização das pesquisas, a próxima etapa foi a definição da string de busca. Algumas palavras-chave relacionadas ao tema da pesquisa foram definidas: aprendizagem móvel, características de qualidade, qualidade de software, métrica de qualidade, modelo de qualidade e avaliação de qualidade. Baseando-se nessas palavras-chave e após testes realizados nas bases de pesquisa, a string de busca selecionada foi:

("m-learning” $\boldsymbol{O R}$ "mobile learning”) $\boldsymbol{A N D}$ ("quality characteristic” $\boldsymbol{O R}$ "quality metric" $\boldsymbol{O R}$ "quality model” $\boldsymbol{O R}$ "software quality" $\boldsymbol{O R}$ "quality evaluation”)

A etapa seguinte consiste na seleção dos estudos primários relevantes. Nesta etapa, a string de busca foi executada nas bases de pesquisa selecionadas. Estas buscas foram realizadas em outubro de 2014 , sendo considerados todos os estudos até esta data. A partir dos resultados obtidos, foram extraídas as informações: título, resumo e palavras-chave. Baseando-se nas informações obtidas, foram aplicados os critérios de inclusão e exclusão que resultaram na seleção preliminar de 19 artigos.

Com a aplicação dos critérios de inclusão e exclusão a seleção de estudos totalizou nove. Além disso, também foram conduzidas pesquisas informais utilizando a string de busca na base de pesquisa Google Scholar, além de pesquisas nas referências dos estudos selecionados. O resultado das buscas informais foi de 14 estudos que, juntamente com as pesquisas anteriores totalizaram 23 estudos, sumarizados na Tabela 3.

\section{Conjunto Preliminar de Características de Qualidade}

Como citado no início desse trabalho, os aspectos de qualidade de uma aplicação $m$ learning é um assunto importante a ser abordado. Sua importância deve-se ao fato de existirem características de qualidade pedagógicas, socioculturais e socioeconômicas, além de características técnicas específicas para o contexto de m-learning.

O mapeamento de um conjunto de características de qualidade para o contexto de $m$-learning se torna-se importante, pois essas características podem ser utilizadas para auxiliar nas etapas de desenvolvimento da aplicação, permitindo que seja feita uma avaliação de qualidade baseada nas características sugeridas. A seguir, uma visão geral das características identificadas é apresentada.

\subsection{Características Técnicas}

Para auxiliar na síntese das informações extraídas relacionadas às características técnicas, as informações foram classificadas de acordo com as características e subcaracterísticas definidas pela norma ISO/IEC 25010 [ISO/IEC 25010 2010].

A Tabela 4 sintetiza as informações relacionadas às características:

- Adequação funcional: refere-se à disponibilidade de funções que atendam as necessidades implícitas e explícitas em relação à aplicação; 
Tabela 3. Resultado da Seleção dos Estudos a partir da Revisão Sistemática

\begin{tabular}{|c|c|}
\hline Ref. & Título \\
\hline [Ali et al. 2012] & A Conceptual Framework for Measuring the Quality Aspects of Mobile Learning \\
\hline [Duarte Filho and Barbosa 2013a] & A contribution to the quality evaluation of mobile learning environments \\
\hline [Parsons and Ryu 2006] & A Framework for Assessing the Quality of Mobile Learning \\
\hline [Duarte Filho and Barbosa 2013b] & A requirements catalog for mobile learning environments \\
\hline [Taylor 2004] & $\begin{array}{l}\text { A task-centred Approach to Evaluating a Mobile Learning Environment for Pe- } \\
\text { dagogical Soundness }\end{array}$ \\
\hline [Boja and Batagan 2009] & Analysis of M-Learning Application Quality \\
\hline [Mostakhdemin-Hosseini 2009] & Analysis of Pedagogical Considerations of M-Learning in Smart Devices \\
\hline [Acharya and Sinha 2013] & Assessing the Quality of M-Learning Systems using ISO/IEC 25010 \\
\hline [Pocatilu et al. 2010] & Development of distributed mobile learning systems \\
\hline [Reis et al. 2012] & Educational Resources for Mobile Wireless Devices: A Case Study \\
\hline [Avellis et al. 2003] & $\begin{array}{l}\text { Evaluating non functional requirements in mobile learning contents and multi- } \\
\text { media educational software }\end{array}$ \\
\hline [Escudeiro and Escudeiro 2012] & $\begin{array}{l}\text { Evaluation of Serious Games in Mobile Platforms with QEF: QEF (Quantitative } \\
\text { Evaluation Framework) }\end{array}$ \\
\hline [Duarte Filho and Barbosa 2012] & $\begin{array}{l}\text { Estudo e Definição de um Conjunto de Características e Requisitos para Ambi- } \\
\text { entes de Aprendizagem Móvel }\end{array}$ \\
\hline [Avellis 2014] & $\begin{array}{l}\text { Mobile Learning and Multimidia Educational Software Requirements' Evalua- } \\
\text { tion }\end{array}$ \\
\hline [Zoran et al. 2012] & Quality Aspects and Metrics in M-Learning Information Systems \\
\hline [Spriestersbach and Springer 2004] & Quality Attributes in Mobile Web Application Development \\
\hline [Pocatilu and Boja 2009] & Quality Characteristics and Metrics related to M-Learning Process \\
\hline [Zamfiroiu 2014] & Quality Metrics for Evaluation Process in M-Learning Environment \\
\hline [Gafni 2009] & Quality Metrics for PDA-based M-Learning Information Systems \\
\hline [Visoiu et al. 2009] & Quality Model for M-Learning Applications \\
\hline [Economides 2008] & Requirements of Mobile Learning Applications \\
\hline [Hujainah et al. 2013] & Usability Guidelines of Mobile Learning Application \\
\hline [Boja et al. 2011] & $\begin{array}{l}\text { Validation of a multi-criteria model used to evaluate M-Learning applications } \\
\text { quality }\end{array}$ \\
\hline
\end{tabular}

- Eficiência do desempenho: verifica o desempenho em relação à quantidade de recursos utilizados sob condições estabelecidas, ou seja, refere-se à otimização do uso dos recursos disponíveis;

- Compatibilidade: refere-se à capacidade da aplicação em trocar informações ou realizar operações com outras aplicações, compartilhando o mesmo ambiente de hardware e/ou software.

Continuando com a síntese das informações encontradas a Tabela 5 apresenta as informações encontradas referentes às características:

- Usabilidade: refere-se à capacidade da aplicação em ser utilizada por usuários específicos em um contexto específico, oferecendo maneiras que permitam que a aplicação seja entendida, aprendida, utilizada e atraente ao usuário;

- Confiabilidade: refere-se à capacidade da aplicação em proporcionar um comportamento consistente com o esperado durante um longo período de tempo;

- Segurança: refere-se à capacidade da aplicação em proteger as informações e dados, protegendo-os de acesso não autorizado e garantido seu acesso de acordo com os diferentes níveis de autorização;

- Portabilidade: refere-se à capacidade da aplicação ser transferida de um hardware ou software para outro.

A característica "Capacidade de Manutenção"não será incluída nos resultados, pois nenhum característica específica para m-learning foi identificada nos estudos. Para esta característica, os estudos apenas citam que elas devem possuir as subcaracterísticas: Modularidade, Reusabilidade, Analisabilidade, Modificabilidade e Testabilidade. 
Tabela 4. Características Técnicas

\begin{tabular}{|c|c|c|}
\hline Característica & Subcaracterística & Descrição \\
\hline \multirow[t]{15}{*}{ Adequação funcional } & Capacidade de comunicação & $\begin{array}{l}\text { Capacidade de transmissão de dados entre dispositi- } \\
\text { vos. }\end{array}$ \\
\hline & Recursos multimídia & $\begin{array}{l}\text { Capacidade de suportar diferentes tipos de mídias, } \\
\text { como vídeo, texto, áudio, entre outros. }\end{array}$ \\
\hline & Completude das características & $\begin{array}{l}\text { Valida se todas as características propostas no projeto } \\
\text { foram de fato implementadas na aplicação. }\end{array}$ \\
\hline & Compactação de mensagem & $\begin{array}{l}\text { Refere-se ao envio de mensagens curtas, provendo } \\
\text { agilidade na comunicação. }\end{array}$ \\
\hline & Precisão & $\begin{array}{l}\text { Capacidade de fornecer resultados exatos e livre de } \\
\text { erros. }\end{array}$ \\
\hline & Concisão das mensagens & $\begin{array}{l}\text { Capacidade das mensagens operacionais da aplicação } \\
\text { serem exatas e de fácil compreensão. }\end{array}$ \\
\hline & Eficácia de execução de tarefas & $\begin{array}{l}\text { Capacidade de facilitar a execução de tarefas, mini- } \\
\text { mizando o tempo, gastos com conexão e quantidade } \\
\text { de dados trafegados. }\end{array}$ \\
\hline & Mudança de contexto & $\begin{array}{l}\text { Comportamento da aplicação em relação a mudança } \\
\text { de contexto. }\end{array}$ \\
\hline & Variação da rede & Comportamento da aplicação ao variar a conexão. \\
\hline & Adaptação às condiçõos físicas & $\begin{array}{l}\text { Capacidade de adaptação da aplicação de acordo com } \\
\text { condições físicas, como ajuste de luz e controle de } \\
\text { ruído. }\end{array}$ \\
\hline & Interface amigável & $\begin{array}{l}\text { A interface com o usuário deve ser amigável, per- } \\
\text { mitindo uma interação agradável entre o usuário e a } \\
\text { aplicação. }\end{array}$ \\
\hline & Carregamento da tela & Quantidade de informações e elementos em uma tela. \\
\hline & Telas por tarefa & $\begin{array}{l}\text { Quantidade de telas utilizadas durante a execução de } \\
\text { uma tarefa. }\end{array}$ \\
\hline & Facilidade de preenchimento & $\begin{array}{l}\text { Capacidade de facilitar o preenchimento de dados } \\
\text { pelo usuário. }\end{array}$ \\
\hline & $\begin{array}{l}\text { Facilidade da saída de dados para o } \\
\text { usuário }\end{array}$ & $\begin{array}{l}\text { Adequação da saída dos dados da aplicação para o } \\
\text { usuário. }\end{array}$ \\
\hline \multirow[t]{9}{*}{ Eficiência do Desempenho } & Transferência de dados & $\begin{array}{l}\text { Refere-se à minimização dos dados, contribuindo as- } \\
\text { sim para a diminuição do tempo de transferência. }\end{array}$ \\
\hline & Tempo de carregamento & $\begin{array}{l}\text { Refere-se ao tempo de espera do usuário até que } \\
\text { ocorra o carregamento completo das informações so- } \\
\text { licitadas. }\end{array}$ \\
\hline & Tempo médio de resposta & $\begin{array}{l}\text { Refere-se à aplicações do tipo cliente-servidor, em } \\
\text { que o tempo de resposta é o tempo entre a requisição } \\
\text { do cliente até o recebimento da resposta pelo cliente. }\end{array}$ \\
\hline & $\begin{array}{l}\text { Tempo de resposta para obter } \\
\text { informação da memória }\end{array}$ & $\begin{array}{l}\text { Tempo de resposta em que a aplicação obtêm } \\
\text { informações armazenadas na memória do dispositivo } \\
\text { móvel. }\end{array}$ \\
\hline & Tamanho da aplicação & $\begin{array}{l}\text { Quantidade de espaço necessário para que a aplicação } \\
\text { possa ser utilizada no dispositivo. }\end{array}$ \\
\hline & Eficiência na utilização de memória & $\begin{array}{l}\text { Implica nas técnicas utilizadas para otimizar o uso da } \\
\text { memória do dispositivo. }\end{array}$ \\
\hline & Eficiência no consumo de energia & $\begin{array}{l}\text { Devido às limitações das baterias dos dispositivos, o } \\
\text { seu uso com eficiência é essencial evitando assim o } \\
\text { desperdício de energia. }\end{array}$ \\
\hline & Limpeza de memória & $\begin{array}{l}\text { Refere-se ao armazenamento de dados desnecessários } \\
\text { no dispositivo, como arquivos que não serão mais uti- } \\
\text { lizados pela aplicação. }\end{array}$ \\
\hline & Escalabilidade & Mede o quanto a aplicação pode ser escalável. \\
\hline \multirow[t]{2}{*}{ Compatibilidade } & Coexistência de aplicação & $\begin{array}{l}\text { Capacidade de várias aplicações serem executadas no } \\
\text { mesmo dispositivo sem ocasionar impactos negativos } \\
\text { de uma aplicação em outra. }\end{array}$ \\
\hline & Expansibilidade & $\begin{array}{l}\text { Capacidade de expansão de uma aplicação por meio } \\
\text { da comunicação com outras aplicações. }\end{array}$ \\
\hline
\end{tabular}

\subsection{Características Pedagógicas}

O aspecto pedagógico de uma aplicação têm por objetivo, abordar as características relacionadas ao ensino e aprendizagem. Este aspecto pode ser dividido em quatro categorias: (i) interatividade, que se refere à interação do usuário com a aplicação; (ii) aprendizagem, que aborda características relacionadas ao aprendizado; (iii) conteúdo, que se refere aos 
Tabela 5. (Cont.) Características Técnicas

\begin{tabular}{|c|c|c|}
\hline Característica & Subcaracterística & Descrição \\
\hline \multirow[t]{11}{*}{ Usabilidade } & Tempo de aprendizado & $\begin{array}{l}\text { Tempo em que o usuário consegue aprender a utilizar } \\
\text { a aplicação. }\end{array}$ \\
\hline & Ajuda & $\begin{array}{l}\text { Capacidade da aplicação em oferecer ajuda para } \\
\text { usuário em relação ao seu uso. }\end{array}$ \\
\hline & Tempo de compreensão & $\begin{array}{l}\text { Tempo gasto para que o usuário compreenda a } \\
\text { aplicação. }\end{array}$ \\
\hline & Homogeneidade do layout & $\begin{array}{l}\text { Relacionada à padronização dos componentes, man- } \\
\text { tendo suas características por todas as telas da } \\
\text { aplicação. }\end{array}$ \\
\hline & Homogeneidade dos componentes & $\begin{array}{l}\text { Relacionada aos diversos tipos de componentes que } \\
\text { possibilitam a mesma funcionalidade, por este motivo } \\
\text { é incentivado a variação mínima de componentes. }\end{array}$ \\
\hline & Entrada de dados & $\begin{array}{l}\text { Em aplicações m-learning não deve-se exigir a en- } \\
\text { trada de muitos dados, já que existem as limitações } \\
\text { de tela e teclado. }\end{array}$ \\
\hline & Navegação & $\begin{array}{l}\text { A aplicação deve ser bem estruturada permitindo fácil } \\
\text { acesso aos conteúdos e atividades. }\end{array}$ \\
\hline & Continuidade & $\begin{array}{l}\text { A aplicação deve ser fluída, não permitindo que o } \\
\text { usuário chegue a um ponto da aplicação onde não } \\
\text { consiga mais navegar por ela. }\end{array}$ \\
\hline & Apresentação de informações & $\begin{array}{l}\text { Capacidade de limitação das informações de acordo } \\
\text { com a área de exibição da tela. }\end{array}$ \\
\hline & Atração/motivação & $\begin{array}{l}\text { A aplicação deve ser atraente e motivante aos usuários } \\
\text { que a utilizarão. }\end{array}$ \\
\hline & Diversidade de dispositivos & $\begin{array}{l}\text { Refere-se à variedade de dispositivos existentes, } \\
\text { permitindo melhorias de usabilidade exclusivas de } \\
\text { acordo com o dispositivo utilizado. }\end{array}$ \\
\hline \multirow[t]{3}{*}{ Confiabilidade } & Acessível & $\begin{array}{l}\text { Refere-se à aplicação estar disponível e acessível a } \\
\text { qualquer hora e em qualquer lugar. }\end{array}$ \\
\hline & Transferência de dados & $\begin{array}{l}\text { Capacidade dos dados serem transferidos, mesmo } \\
\text { ocorrendo desconexões, ainda que a transferência não } \\
\text { seja possível, a aplicação deve garantir que os dados } \\
\text { não serão perdidos. }\end{array}$ \\
\hline & $\begin{array}{l}\text { Tempo de recuperação após desco- } \\
\text { nexão }\end{array}$ & $\begin{array}{l}\text { Capacidade da aplicação em restabelecer a conexão } \\
\text { após uma desconexão, devendo ainda manter o seu } \\
\text { funcionamento devido a estas interrupções inespera- } \\
\text { das. }\end{array}$ \\
\hline \multirow[t]{8}{*}{ Segurança } & Acesso & Capacidade em prover acesso seguro e privado. \\
\hline & Criptografia & $\begin{array}{l}\text { Utilizando criptografia nos dados sensíveis que são } \\
\text { trafegados e armazenados pela aplicação. }\end{array}$ \\
\hline & $\begin{array}{l}\text { Mensagens seguras e informações } \\
\text { no dispositivos }\end{array}$ & $\begin{array}{l}\text { Refere-se à utilização de mecanismos de segurança, } \\
\text { como identificação, autorização e confidencialidade. }\end{array}$ \\
\hline & Confiabilidade & $\begin{array}{l}\text { Capacidade de prover acesso apenas para usuários e } \\
\text { aplicações autorizadas. }\end{array}$ \\
\hline & $\begin{array}{l}\text { Permitir salvar pontos de backup } \\
\text { em caso de bateria baixa }\end{array}$ & $\begin{array}{l}\text { Deve salvar pontos de backup em caso de bate- } \\
\text { ria baixa, podendo ser automática ou sugerindo a } \\
\text { execução da ação para o usuário. }\end{array}$ \\
\hline & Rastreabilidade & $\begin{array}{l}\text { Refere-se ao armazenamento dos registros de eventos } \\
\text { ocorridos na aplicação, sendo possível assim rastrear } \\
\text { os caminhos realizados pelo usuário. }\end{array}$ \\
\hline & Autenticidade & $\begin{array}{l}\text { Refere-se à garantia de autenticidade das ações do } \\
\text { usuário. }\end{array}$ \\
\hline & Utilização de perfil de usuário & $\begin{array}{l}\text { Deve-se utilizar diferentes perfis de usuário, restrin- } \\
\text { gindo assim o acesso a determinadas funcionalidades } \\
\text { do sistema. }\end{array}$ \\
\hline \multirow[t]{4}{*}{ Portabilidade } & Adaptação de dispositivos & $\begin{array}{l}\text { Capacidade da aplicação em se adaptar a diferentes } \\
\text { tipos de dispositivos móveis. }\end{array}$ \\
\hline & Sucesso de instalação & $\begin{array}{l}\text { Capacidade da aplicação ser instalada e desinstalada } \\
\text { em diferentes dispositivos móveis sem implicar em } \\
\text { erros. }\end{array}$ \\
\hline & Facilidade de instalação & $\begin{array}{l}\text { Refere-se à facilidade e à velocidade com que a } \\
\text { aplicação é instalada em um dispositivo. }\end{array}$ \\
\hline & Atualização e configuração & $\begin{array}{l}\text { A aplicação deve ser atualizada e configurada de ma- } \\
\text { neira rápida e automática. }\end{array}$ \\
\hline
\end{tabular}


materiais utilizados nas aplicações; e (iv) aplicação, que são características técnicas da aplicação que podem interferir nas questões pedagógicas. As características identificas são sintetizadas na Tabela 6 .

\subsection{Características Socioeconômicas}

O aspecto socioeconômico de uma aplicação é composto por características relacionadas à viabilidade da aplicação, fazendo uma relação de custo e benefício. Referente a este aspecto, foram identificadas as seguintes características:

- Custo para desenvolver o material de aprendizagem [Zoran et al. 2012]: os gastos com o desenvolvimento do material de aprendizagem influenciam diretamente os gastos com a aplicação e se o seu custo for repassado para os usuário, implica em um aumento no investimento por parte do mesmo.

- Tempo para desenvolver o material de aprendizagem [Zoran et al. 2012]: esta característica causa impacto direto na anterior, pois um maior tempo de desenvolvimento pode acarretar em um aumento nos custos e em atrasos na disponibilização do material para os aprendizes.

- Nível de serviço [Duarte Filho and Barbosa 2013b, Economides 2008]: o estabelecimento de níveis de serviço possibilita maior segurança na utilização das aplicações e permite existir continuidade no seu uso. Para isso, as seguintes subcaracterísticas foram identificadas: (i) plano de continuidade: deve-se possuir estratégias e procedimentos de recuperação dos ambientes m-learning, evitando que as práticas de aprendizagem sejam interrompidas; (ii) acordo de nível de serviço: é necessário possuir acordo de nível de serviço, devendo este ser baseado no número de participantes, quantidade de materiais e funcionalidades da aplicação; e (iii) custo-benefício: a aplicação deve ser economicamente viável. Por este motivo, os custos de desenvolvimento, aquisição e manutenção devem ser baixos.

- Suporte [Duarte Filho and Barbosa 2013b, Economides 2008]: os serviços oferecidos pela aplicação m-learning devem possuir um suporte eficiente aos usuários. Relacionados a isso, dois pontos são considerados importantes: (i) qualquer mudança ou ação no ambiente m-learning deve ser adaptada para o usuário final de acordo com seu perfil, preferências e restrições; e (ii) as respostas a qualquer mudança (física ou ambiental) devem ser tratadas de forma clara.

\subsection{Características Socioculturais}

$\mathrm{O}$ aspecto sociocultural tem como objetivo garantir qualidade com relação às características socioculturais dos usuários ou local onde a aplicação será utilizada. Este aspecto considera características como, idade do usuário, nível de ensino, determinado grupo cultural, entre outros. As características socioculturais identificadas no estudos foram:

- Curva de aprendizado [Pocatilu and Boja 2009]: refere-se ao tempo em que o usuário consegue manusear a tecnologia móvel, ou seja, está diretamente relacionada à tecnologia dos dispositivos móveis.

- Idade do usuário [Pocatilu and Boja 2009]: de acordo com a idade dos usuários, diferentes limitações podem existir. Por este motivo, é necessário que a aplicação seja adaptável. 
Tabela 6. Características Pedagógicas

\begin{tabular}{|c|c|c|}
\hline Categoria & Característica & Descrição \\
\hline \multirow[t]{2}{*}{ Interatividade } & Feedback & $\begin{array}{l}\text { Capacidade da aplicação em permitir o feedback recíproco en- } \\
\text { tre alunos e professores. }\end{array}$ \\
\hline & Interação entre usuários & $\begin{array}{l}\text { A aplicação deve permitir a interação entre seus usuários, po- } \\
\text { dendo prover este recurso através de blogs, fóruns, grupos, en- } \\
\text { tre outros. }\end{array}$ \\
\hline \multirow[t]{10}{*}{ Aprendizagem } & Identificação de deficiências & $\begin{array}{l}\text { Capacidade de auxiliar na identificação de lacunas de conheci- } \\
\text { mento. }\end{array}$ \\
\hline & Identificação de perfis & $\begin{array}{l}\text { Deve ser possível a identificação dos diferentes perfis de aluno } \\
\text { de acordo com suas características, como idade, nível de co- } \\
\text { nhecimento, entre outros. }\end{array}$ \\
\hline & $\begin{array}{l}\text { Processos educativos e de aprendi- } \\
\text { zagem definidos }\end{array}$ & $\begin{array}{l}\text { Os processos educacionais e de aprendizagem utilizados na } \\
\text { aplicação devem ser bem definidos. }\end{array}$ \\
\hline & Ambiente de aprendizagem & $\begin{array}{l}\text { Capacidade de prover um ambiente que favoreça a aprendiza- } \\
\text { gem do aluno, provendo maneiras para manter o interesse do } \\
\text { aluno. }\end{array}$ \\
\hline & Ganho de conhecimento & $\begin{array}{l}\text { Deve prover um sistema de avaliação com o objetivo de verifi- } \\
\text { car se a aplicação está provendo ganho de conhecimento para } \\
\text { o usuário. }\end{array}$ \\
\hline & Conhecimento na hora certa & $\begin{array}{l}\text { O conteúdo deve ser oferecido na hora correta, evitando ofe- } \\
\text { recer um conteúdo para ensinar algo que dependa de um } \\
\text { conteúdo ainda não estudado pelo usuário. }\end{array}$ \\
\hline & Atividades educacionais & $\begin{array}{l}\text { A aplicação deve oferecer diferentes tipos de atividades rela- } \\
\text { cionadas à educação, provendo assim a aquisição de conhe- } \\
\text { cimento e a possibilidade do aluno receber orientação de um } \\
\text { tutor nas atividades. }\end{array}$ \\
\hline & Adaptação ao contexto & $\begin{array}{l}\text { A aplicação deve ser capaz de se adaptar ao contexto do } \\
\text { usuário, por exemplo, adaptar-se à um usuário do meio rural, } \\
\text { com idade adulta e com escolaridade baixa. }\end{array}$ \\
\hline & $\begin{array}{l}\text { Iniciativa de aquisição de conheci- } \\
\text { mento }\end{array}$ & $\begin{array}{l}\text { A aplicação deve estimular o usuário a tomar a iniciativa em } \\
\text { relação a aquisição de conhecimento, permitindo que o usuário } \\
\text { decida sobre quais tarefas deseja realizar. }\end{array}$ \\
\hline & Atividades de instrução & $\begin{array}{l}\text { Refere-se ao fornecimento de instruções para guiar o usuário } \\
\text { durante a aprendizagem. }\end{array}$ \\
\hline \multirow[t]{9}{*}{ Conteúdo } & Qualidade de conteúdo & $\begin{array}{l}\text { A aplicação deve prover conteúdo com qualidade, além de ser } \\
\text { relacionado com situações e problemas de interesse do aluno, } \\
\text { o conteúdo não deve possuir erros ortográficos, mensagens in- } \\
\text { vasivas, negativas e com conteúdo de discriminação racial ou } \\
\text { religiosa. }\end{array}$ \\
\hline & Usuário alvo & $\begin{array}{l}\text { Refere-se à definição dos tipos de usuários que a aplicação pre- } \\
\text { tende atingir. }\end{array}$ \\
\hline & Complexidade multimídia & $\begin{array}{l}\text { A aplicação não deve possuir muitos elementos multimídias, } \\
\text { pois estes podem ocasionar em pontos de distração para o } \\
\text { usuário. }\end{array}$ \\
\hline & Fonte de conteúdo & $\begin{array}{l}\text { Todo material disponibilizado pela aplicação deve ser de fonte } \\
\text { confiável }\end{array}$ \\
\hline & Gerenciamento de conteúdo & $\begin{array}{l}\text { Refere-se ao controle, gerenciamento e monitoramento dos } \\
\text { conteúdos oferecidos. }\end{array}$ \\
\hline & Integração de conteúdo & $\begin{array}{l}\text { Refere-se à integração dos conteúdos, provendo um fluxo defi- } \\
\text { nido entre eles. }\end{array}$ \\
\hline & Recursos didáticos & $\begin{array}{l}\text { Refere-se aos recursos didáticos oferecidos pela aplicação para } \\
\text { auxiliar o aprendizado. }\end{array}$ \\
\hline & $\begin{array}{l}\text { Reutilização de conteúdo de apren- } \\
\text { dizagem }\end{array}$ & Capacidade da aplicação em prover o reuso de conteúdo. \\
\hline & Complexidade de conteúdo & $\begin{array}{l}\text { Os conteúdos não devem possuir excesso de informações nas } \\
\text { lições, devendo ser oferecido gradativamente, evitando assim } \\
\text { que um conteúdo complexo possa afetar a compreensão do } \\
\text { usuário. }\end{array}$ \\
\hline \multirow[t]{3}{*}{ Aplicação } & Visualizações separadas & $\begin{array}{l}\text { Refere-se à separação das visões do aluno e tutor, ou seja, } \\
\text { de acordo com cada perfil de usuário a aplicação deve prover } \\
\text { visões diferentes. }\end{array}$ \\
\hline & Esforço cognitivo das atividades & $\begin{array}{l}\text { Refere-se ao esforço cognitivo exercido pelo usuário na } \\
\text { utilização da aplicação e na aquisição de conhecimento. }\end{array}$ \\
\hline & Qualidade audiovisual & $\begin{array}{l}\text { A aplicação deve prover a aprendizagem utilizando mídias com } \\
\text { qualidade. }\end{array}$ \\
\hline
\end{tabular}


- Satisfação do usuário [Pocatilu and Boja 2009, Hujainah et al. 2013]: a fim de suprir as expectativas do usuário, a aplicação deve satisfazê-lo, evitando assim abandono na utilização da aplicação.

- Colaboração [Duarte Filho and Barbosa 2013b, Economides 2008]: a colaboração deve estar presente nas aplicações $m$-learning, permitindo que os aprendizes e tutores se relacionem. A colaboração pode ser proporcionada por meio de wiki, jogos, microblogs, fóruns e grupos.

- Atividades individuais e em grupos [Economides 2008]: as aplicações m-learning devem oferecer atividades individuais e em grupo. As atividades proporcionam a possibilidade de se aprender individualmente, mas também motivam o desenvolvimento de tarefas em conjunto.

\subsection{Características dos Dispositivos Móveis}

Para diminuir os impactos que os dispositivos móveis possam exercer sobre uma aplicação m-learning, é necessário conhecer as características dos dispositivos móveis que possam afetar a qualidade das aplicações m-learning. A fim de organizar estas características, elas foram divididas em duas categorias foram consideradas: (i) comunicação, que são as características que compõem a comunicação do dispositivo, por exemplo, a infraestrutura de rede; e (ii) hardware, que está relacionada aos componentes físicos dos dispositivos.

Referente à categoria comunicação, foram encontradas as características: (i) banda estreita (referente à largura da banda disponível), sendo necessário adaptar a aplicação de acordo com a banda, diminuindo a sua utilização, como por exemplo, o uso de texto ao invés de imagens; (ii) falta de cobertura, ou seja, as operadoras de telefonia possuem falhas em sua cobertura; e (iii) instabilidade de conexão, que se refere à perda de conexão e variações de velocidade de conexão.

Com relação à categoria hardware, as características encontradas foram: (i) teclado pequeno; (ii) tela pequena; (iii) memória com pouca capacidade; (iv) baixo poder de processamento; (v) baixa autonomia da bateria; e (v) diversidade de dispositivos.

\subsection{Catálogo de Características}

A definição de um conjunto de características relacionado à um contexto específico, ajuda não somente na modelagem, padronização e manutenção, mas também na garantia de qualidade e na eficiência do sistema [Duarte Filho and Barbosa 2012]. No cenário emergente em que se encontra m-learning, a qualidade não é apenas relacionada à aspectos técnicos. Há também uma necessidade de lidar com questões intrínsecas (por exemplo, educacional, sociocultural e econômica) relacionadas com as atividades diárias de aprendizes e tutores [Economides 2008].

Diante deste cenário e os resultados obtidos com a revisão sistemática, um catálogo de características de qualidade para aplicações educacionais móveis foi proposto. Um "mapa mental", apresentado na Figura 1, foi criado contendo as características e requisitos relevantes ao contexto de m-learning.

\section{Conclusões e Trabalhos Futuros}

Este trabalho teve como principal objetivo identificar um conjunto de características de qualidade para aplicações m-learning, considerando os aspectos técnicos, pedagógicos, 


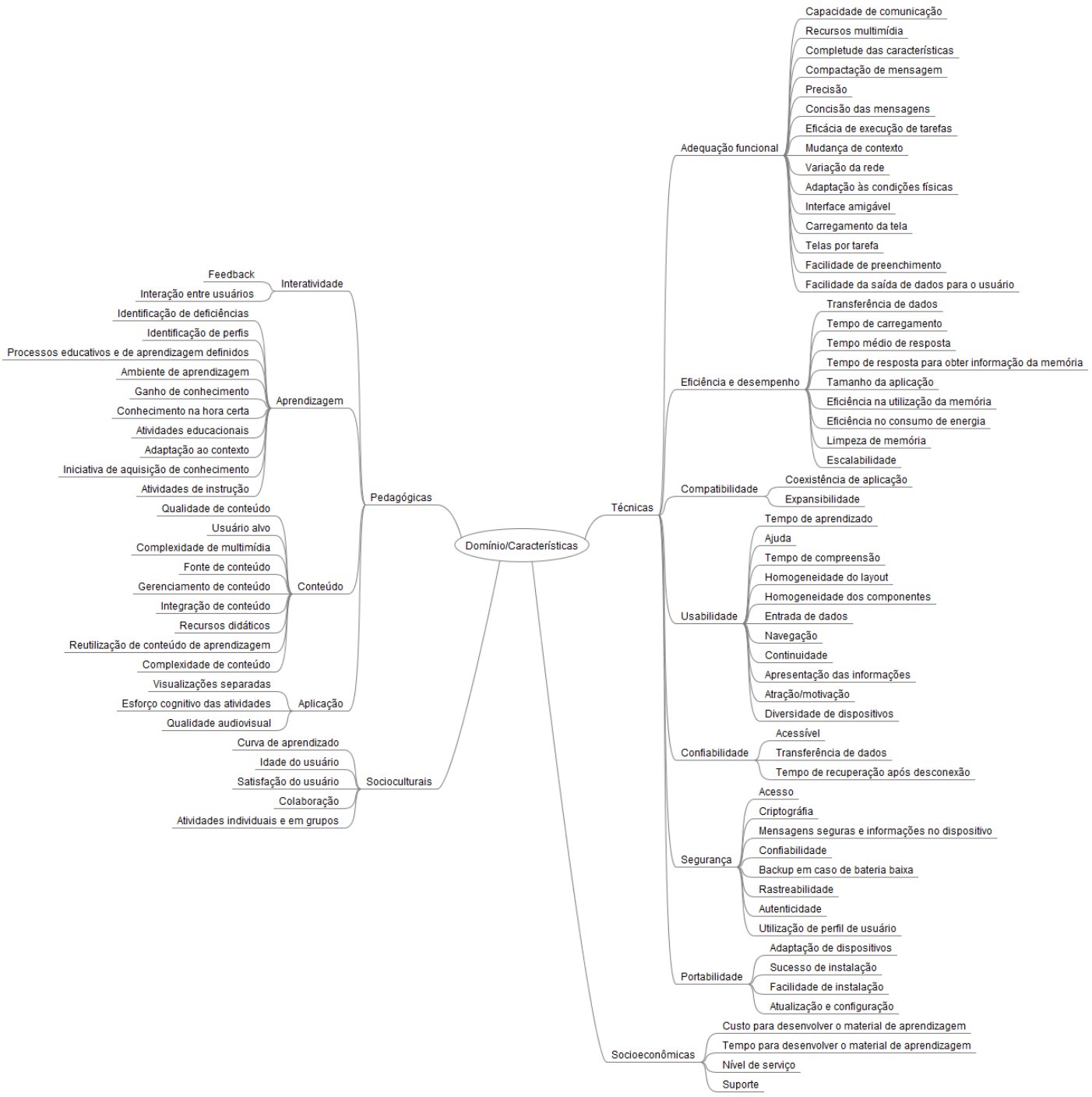

Figura 1. Mapa Mental: Características para M-Learning

socioeconômicos e socioculturais, além de identificar também as características dos dispositivos móveis que possam influenciar na qualidade de uma aplicação $m$-learning.

As características técnicas encontradas estão relacionadas às tecnologias de hardware e software envolvidas em uma aplicação de aprendizagem móvel. Já as características pedagógicas estão relacionadas às questões educacionais e as socioculturais, aos aspectos culturais que envolvem os utilizadores desse tipo de aplicação. Por fim, as características socioeconômicas envolvem os impactos econômicos e sociais.

Para identificar as características foi realizada uma revisão sistemática que resultou no total, 23 estudos. Por este motivo, pôde-se concluir que existem poucos estudos relacionados à qualidade de aplicações $m$-learning. Embora a quantidade de estudos seja pequena, foi possível identificar várias características de qualidade para aplicações $m$ learning. 
A principal contribuição desse estudo é o conjunto de características identificadas que foram consolidadas em um catálogo. O catálogo proposto pode ser utilizado para auxiliar no desenvolvimento de um modelo de qualidade que, por sua vez, poderá ser utilizado na avaliação de qualidade de aplicações $m$-learning, além de auxiliar na concepção de aplicações desse tipo. A partir dos resultados obtidos também é possível concluir que existem muitos desafios para que os desenvolvedores de aplicações $m$-learning consigam satisfazer todas ou ao menos parte das características propostas no catálogo.

Além do catálogo proposto, também foram apresentadas, características dos dispositivos móveis que podem afetar diretamente a qualidade de uma aplicação $m$-learning . A identificação dessas características é importante, pois deve-se conhecê-las, visando adequar a aplicação para diminuir os seus impactos.

Para trabalhos futuros, os autores pretendem evoluir e melhorar o conjunto de características proposto, proporcionando um conjunto de requisitos de qualidade específicos para aplicações m-learning. Tal conjunto deve ser estruturado em critérios, requisitos e descrições de qualidade, servindo de guia para as organizações desenvolvedoras, garantindo adequação de qualidade e satisfação às necessidades educacionais e de aprendizagem.

\section{Referências}

Acharya, A. and Sinha, D. (2013). Assessing the quality of m-learning systems using ISO/IEC 25010.

Al-Ani, M., Hameed, S., and Faisal, L. (2013). Students' perspectives in adopting mobile learning at university of bahrain. In e-Learning "Best Practices in Management, Design and Development of e-Courses: Standards of Excellence and Creativity", 2013 Fourth International Conference on, pages 86-89.

Ali, A., Ouda, A., and Capretz, L. F. (2012). A conceptual framework for measuring the quality aspects of mobile learning. Bulletin of the IEEE Technical Committee on Learning Technology, 14(4):31. 02.

Avellis, G. (2014). Mobile learning and multimedia educational software requirements' evaluation. International Journal of Education and Information Technologies, 8:8392.

Avellis, G., Scaramuzzi, A., and Finkelstein, A. (2003). Evaluating non functional requirements in mobile learning contents and multimedia educational software.

Barbosa, E. F. (2004). Uma contribuição ao processo de desenvolvimento e modelagem de módulos educacionais. PhD thesis, Universidade de São Paulo.

Boja, C. and Batagan, L. (2009). Analysis of m-learning applications quality. W. Trans. on Comp., 8(5):767-777.

Boja, C., Bătăgan, L., and Vişoiu, A. (2011). Validation of a multi-criteria model used to evaluate m-learning applications quality. In Proceedings of the 12th International Conference on Computer Systems and Technologies, CompSysTech '11, pages 590595, New York, NY, USA. ACM.

Duarte Filho, N. F. and Barbosa, E. F. (2012). Estudo e definicao de um conjunto de caracteristicas e requisitos para ambientes de aprendizagem movel. In SBIE, 2012. 
Duarte Filho, N. F. and Barbosa, E. F. (2013a). A contribution to the quality evaluation of mobile learning environments. In Frontiers in Education Conference, 2013 IEEE, pages $379-382$.

Duarte Filho, N. F. and Barbosa, E. F. (2013b). A requirements catalog for mobile learning environments. In Proceedings of the 28th Annual ACM Symposium on Applied Computing, SAC '13, pages 1266-1271, New York, NY, USA. ACM.

Economides, A. A. (2008). Requirements of Mobile Learning Applications. International Journal of Innovation and Learning, 5.

Escudeiro, P. and Escudeiro, N. (2012). Evaluation of serious games in mobile platforms with qef: Qef (quantitative evaluation framework). In Wireless, Mobile and Ubiquitous Technology in Education (WMUTE), 2012 IEEE Seventh International Conference on, pages 268-271.

Fazlina, S., Manap, A., and Rias, R. (2013). Mobile learning awareness among students at higher learning institutes: A case study. In Informatics and Creative Multimedia (ICICM), 2013 International Conference on, pages 226-229.

Gafni, R. (2009). Quality metrics for pda-based m-learning information systems. Interdisciplinary Journal of E-Learning and Learning Objects, 5(1):359-378.

Huang, Y.-M., Lin, Y.-T., and Cheng, S.-C. (2010). Effectiveness of a mobile plant learning system in a science curriculum in taiwanese elementary education. Computers \& Education, 54(1):47-58.

Hujainah, F., Dahlan, H., and Al-haimi, B. (2013). Usability guidelines of mobile learning application. Journal of Information Systems Research and Innovation.

ISO/IEC 25010 (2010). ISO/IEC 25010 - Systems and software engineering - Systems and software Quality Requirements and Evaluation (SQuaRE) - System and software quality models. Technical report.

ISO/IEC 8402, . (1994). ISO 8402: Quality Management and Quality Assurance : Vocabulary. ISO/IEC.

ISO/IEC 9126, . (2001). ISO/IEC 9126. Software engineering - Product quality. ISO/IEC.

Kinshuk, S. J., Sutinen, E., and Goh, T. (2003). Mobile technologies in support of distance learning. Asian Journal of Distance Education, 1(1):60-68.

Kitchenham, B. and Charters, S. (2007). Guidelines for performing Systematic Literature Reviews in Software Engineering. Technical Report EBSE 2007-001, Keele University and Durham University Joint Report.

Mishra, N. and Chavhan, R. (2012). Effectiveness of mobile learning on awareness about learning disability among student teachers. In Technology Enhanced Education (ICTEE), 2012 IEEE International Conference on, pages 1-6.

Mostakhdemin-Hosseini, A. (2009). Analysis of pedagogical considerations of mlearning in smart devices. iJIM, 3(4):33-34.

Nah, K. C., White, P., and Sussex, R. (2008). The potential of using a mobile phone to access the internet for learning efl listening skills within a korean context. ReCALL, 20(03):331-347. 
O’Malley, C., Vavoula, G., Glew, J., Taylor, J., Sharples, M., and Lefrere, P. (2003). Guidelines for learning/teaching/tutoring in a mobile environment. mobilearn project deliverable. Retrieved March, 24:2013.

Parsons, D. and Ryu, H. (2006). A framework for assessing the quality of mobile learning. In International Conference for Process Improvement, Research and Education, pages $17-27$.

Picek, R. and Grcic, M. (2013). Evaluation of the potential use of m-learning in higher education. In Information Technology Interfaces (ITI), Proceedings of the ITI 2013 35th International Conference on, pages 63-68.

Pocatilu, P. and Boja, C. (2009). Quality Characteristics and Metrics related to MLearning Process. The Amfiteatru Economic journal, 11(26):346-354.

Pocatilu, P., Doinea, M., and Ciurea, C. (2010). Development of distributed mobile learning systems. In Proceedings of the 9th WSEAS International Conference on Circuits, Systems, Electronics, Control \&\#38; Signal Processing, CSECS '10, pages 196-201, Stevens Point, Wisconsin, USA. World Scientific and Engineering Academy and Society (WSEAS).

Pressman, R. (2010). Software Engineering: A Practitioner's Approach. McGraw-Hill higher education. McGraw-Hill Education.

Reis, R., Escudeiro, P., and Escudeiro, N. (2012). Educational resources for mobile wireless devices: A case study. In Wireless, Mobile and Ubiquitous Technology in Education (WMUTE), 2012 IEEE Seventh International Conference on, pages 264-267.

Soad, G., Duarte Filho, N., and Barbosa, E. (2015). Revisão sistemática sobre características de qualidade de aplicações educacionais móveis: Relatório técnico (em elaboração). Technical report.

Spriestersbach, A. and Springer, T. (2004). Quality attributes in mobile web application development.

Svetlana, K. et al. (2009). Adaptation e-learning contents in mobile environment. In Proceedings of the 2nd International Conference on Interaction Sciences: Information Technology, Culture and Human, pages 474-479. ACM.

Taylor, J. (2004). A task-centred approach to evaluating a mobile learning environment for pedagogical soundness. pages 167-171+.

Visoiu, A., Batagan, L., and Boja, C. (2009). Quality model for m-learning applications. In Proceedings of the 8th WSEAS International Conference on Data Networks, Communications, Computers, DNCOCO'09, pages 60-66, Stevens Point, Wisconsin, USA. World Scientific and Engineering Academy and Society (WSEAS).

Wexler, S., Brown, J., Metcalf, D., Rogers, D., and Wagner, E. (2008). Mobile learning: What it is, why it matters, and how to incorporate it into your learning strategy. Guild Research.

Zamfiroiu, A. (2014). Quality metrics for evaluation process in m-learning environment. European Scientific Journal, 1.

Zoran, K., Arsovski, S., Arsovski, Z., Rankovic, V., Milanovic, I., and Rejman-Petrovic, D. (2012). Quality aspects and metrics in m-learning information systems. 